\title{
Kinetics of Reduction of Iron Oxide Pellets with Hydrogen at Low Temperatures*
}

\author{
By Yukiaki Hara** and Shin-ichi Kondō ${ }^{* *}$
}

\begin{abstract}
Synopsis
An analytical method of experimental data for the reduction of ironoxide pellets has been developed under the consideration of rate determining mechanism which combines the intraparticle gaseous diffusion through the reduced layer with the chemical reaction at the interface between the reduced and unreduced layers. In this method, the assumption that the reduction proceeds topochemically, and the times required for 50 and $87.5 \%$ reduction have been adopted. It was also found that this method might be applied to the case in which the adsorption term of water vapor was not neglected.

Porous pellets prepared from the pulverized Brazilian ore, high quality hematite, were reduced with hydrogen or hydrogen diluted with nitrogen at low temperatures $\left(500^{\circ}\right.$ and $550^{\circ} \mathrm{C}$ ) and the decreases in weight were measured with a thermobalance. Data obtained were analyzed by the above method, and the effects of the reducing temperature, the diluent gas, the porosity of pellet upon the intraparticle diffusivity and the chemical reaction constant were examined.
\end{abstract}

\section{Introduction}

The overall reaction rate in the gaseous reduction of an iron-oxide pellet is usually determined by the resistances of the following three steps; the diffusion through the gaseous film around the pellet, the intraparticle diffusion through the reduced layer and the chemical reaction at the interface between the reduced and unreduced layers. However, if the gas velocity is large enough, the resistance of gas film may be eliminated, then the reaction will proceed with the combined rate determining mechanism of the intraparticle duffusion and the chemical reaction. In the studies on the reduction kinetics of iron oxide pellets, it has formerly been considered that the rate determining step was either the intraparticle diffusion ${ }^{1,2)}$ or the chemical reaction. ${ }^{3), 4)}$ However, some investigators ${ }^{5)-13)}$ recently proposed that the experimental data should be analyzed in terms of the combined rate determining mechanism of the above mentioned two steps.

Several investigators observed that the existence of water vapor retarded the reaction by the adsorption on reacting surface when the iron oxides were reduced with hydrogen at low temperature below $570^{\circ}$ C. ${ }^{13)-17)}$ If one considers the effect of adsorption in the combined rate determining mechanism, the rate equation for the reduction of pellets becomes more complicated and the analysis of experimental data can hardly be made. ${ }^{8), 13)}$

A simple method of analysis for the combined rate determining mechanism was therefore devised. By this method, the intraparticle diffusion coefficient and the chemical reaction constant are easily calculated from the times required for 50 and $87.5 \%$ reduction in the case that the adsorption term is not neglected.

Porous iron-oxide pellets prepared from the Brazilian ore, high quality hematite, were reduced with hydrogen or hydrogen diluted with nitrogen at low temperatures $\left(500^{\circ}\right.$ and $550^{\circ} \mathrm{C}$ ) and the changes in weight were measured with a thermobalance. Data obtained have been analyzed by the above-mentioned method. In addition, the effects of the diluent gas, the pellet porosity for the intraparticle diffusivity and the chemical reaction constant have been examined.

This report is the summarization of three papers published in Japanese ${ }^{18)-20)}$ on the reduction of ironoxide pellets with hydrogen at low temperatures.

\section{Method of Analysis by the Combined Rate Determining Mechanism of Intraparticle Diffusion and Chemical Reaction}

Assuming the topochemical reaction for the reduction of a spherical iron-oxide pellet, the reduction rate in the rate determining mechanism which combines the intraparticle diffusion through the reduced layer with the chemical reaction at the interface is given as follows :

Adopting the quasi-steady state $^{21)}$ for the intraparticle diffusion, the gaseous diffusion rate $V_{D}$ is

$$
V_{D}=\frac{4 \pi r_{o} r_{i}}{r_{o}-r_{i}} D_{e} \cdot\left(C_{i}-C_{o}\right)
$$

The chemical reaction rate $V_{C}$ at the interface is

$$
V_{C}=4 \pi r_{i}^{2} k_{c}(1+1 / K) \cdot\left(C_{e}-C_{i}\right) \cdot f\left(C_{i}\right)
$$

where $f\left(C_{i}\right)$ is the adsorption term.

Since $V_{D}=V_{C}=V$ under the steady state and $V$ may be converted to the decreasing rate of the radius of reaction interface, the generalized rate equation is expressed by

$$
\begin{aligned}
V & \equiv-\frac{4 \pi r_{i}^{2}\left(\begin{array}{c}
d r_{i} \\
d \theta
\end{array}\right)}{} \\
& \left.=\frac{4 \pi r_{o}^{2}\left(C_{e}-C_{o}\right)}{\left\{\left(\begin{array}{c}
r_{o} \\
r_{i}
\end{array}\right)^{2} \cdot k_{c}(1+1 / K) \cdot f\left(C_{i}\right)\right.}+\begin{array}{c}
1 \\
r_{o}\left(r_{o}-r_{i}\right) \\
r_{i}
\end{array} \cdot \begin{array}{c}
1 \\
D_{e}
\end{array}\right\}
\end{aligned}
$$

where $\alpha$ is the equivalent volume of the reducing gas for the pellet.

Each term in the denominator of right-hand side of Eq. (3) represents the resistances of chemical reaction and intraparticle diffusion, respectively. Therefore, the ratio between these two resistances is 


$$
\frac{\lambda_{D}}{\lambda_{C}}=\left(1-\frac{r_{i}}{r_{o}}\right)\left(\frac{r_{i}}{r_{o}}\right) \frac{r_{o} k_{c}(1+1 / K)}{D_{e}} \cdot f\left(C_{i}\right)
$$

On the other hand, the water concentration at the interface $C_{i}$ is given by

$$
\begin{aligned}
& C_{i}-C_{o} \\
& C_{e}-C_{i}
\end{aligned}=\left(1-\frac{r_{i}}{r_{o}}\right)\left(\frac{r_{i}}{r_{o}}\right) \cdot \frac{r_{o} k_{c}(1+1 / K)}{D_{e}} \cdot f\left(C_{i}\right)
$$

Here, the adsorption term $f\left(C_{i}\right)$ is a function of the water concentration $C_{i}$, and the values of $f\left(C_{i}\right)$ are $f\left(C_{i}\right)=1.0$ at $C_{i}=0$ and $f\left(C_{i}\right)<1.0$ at $C_{i}>0$. This function is obtained from the experimental data reported by the other investigators. ${ }^{13)-17)}$ If $f\left(C_{i}\right)$ is taken into account, Eq. (3) becomes non-linear, so that the integrated form is not obtained analytically.

Now, if the adsorption term is neglected, that is, the value of $f\left(C_{i}\right)$ is always unity, the integrated form of the rate equation is given as follows:

$$
\begin{aligned}
\theta= & \frac{r_{o}}{\alpha k_{c}(1+1 / K) \cdot\left(C_{e}-C_{o}\right)}\left\{1-(1-R)^{1 / 3}\right\} \\
& +\underset{6 \alpha D_{e}\left(C_{e}-C_{o}\right)}{ } \cdot\left\{3-3(1-R)^{2 / 3}-2 R\right\}
\end{aligned}
$$

Hence, substituting $R=1.0$ in Eq. (6), the time required for complete reduction $\theta_{0}$ is

$$
\theta_{0}=\frac{r_{o}^{2}}{6 \alpha\left(C_{e}-C_{o}\right)}\left\{\begin{array}{c}
6 \\
k_{c}(1+1 / K) r_{o}
\end{array}+\frac{1}{D_{e}}\right\}
$$

From Eqs. (6) and (7), the dimensionless reaction time difined as $\tau \equiv \theta \mid \theta_{0}$ is expressed as follows :

$$
\tau=\frac{(6 / \gamma)\left\{1-(1-R)^{1 / 3}\right\}+\left\{3-3(1-R)^{2 / 3}-2 R\right\}}{1+(6 / \gamma)} .
$$

where

$$
\gamma=r_{o} k_{c}(1+1 / K) / D_{e}
$$

Here, $\gamma$ is related to the resistance ratio between the intraparticle diffusion and the chemical reaction, so $\gamma$ is named "resistance ratio". In the extreme case that only the chemical reaction controls, the value of $\gamma$ is equal to zero ; on the other hand, only the intraparticle diffusion controls, the value of $\gamma$ becomes infinity. The relationships between the fractional reduction $R$ and $\tau$ for the cases of reaction control and of diffusion control are shown in Fig. 1. In the combined rate

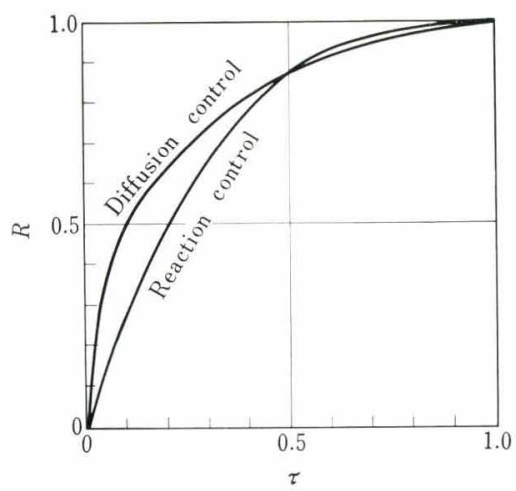

Fig. 1. Dimensionless reaction time $\tau \equiv \theta \mid \theta_{0}$ is. fractional reduction $R$ control, the curve of $R$ vs. $\tau$ is located in the position between these two curves in this figure which is determined by the value of $\gamma$. That is, the relative value of $\tau$ is given by the following equation independently of the degree of reduction.

$$
Z=\frac{\tau-\tau_{D}}{\tau_{C}-\tau_{D}}=\frac{6}{6+\gamma}
$$

where $\tau_{C}$ and $\tau_{D}$ represent $\tau$ for the chemical reaction and the diffusion controls.

From Eq. (8), $\tau$ at $R=0.875$ is always

$$
\tau_{87.5}=0.500
$$

and $\tau$ at $R=0.500$ is

$$
\tau_{50}=(0.1101 \gamma+1.238) /(6+\gamma)
$$

The relative value defined as $\zeta \equiv \theta_{0} / \theta_{0 . C}$ is

$$
\zeta=1+(\gamma / 6)
$$$$
\text { where } \quad \theta_{0 \cdot C}=r_{o} / \alpha k_{c}(1+1 / K)\left(C_{e}-C_{o}\right)
$$

In the above equation, $\theta_{0 . C}$ represents the time required for complete reduction in the case of reaction control. By using Eqs. (11) to (14), the intraparticle diffusion coefficient $D_{e}$ and the chemical reaction constant $k_{c}$ may easily be calculated from the times required for 50 and $87.5 \%$ reduction.

Now, let us consider the case in which the adsorption term should be accounted. Since Eq. (3) shows a nonlinear form, its integration have to be made by the numerical calculation. When the results of the integration are overlooked, the values of $R$ are always equal to 0.875 at $\tau=0.50$ in the relation between the dimentionless time, $\tau$, and the degree of reduction, $R$, because the value of the denominator in Eq. (3), that is the resistances for reduction, is symmetrical to $\left(r_{i} / r_{o}\right)=$ 0.50 .

If the relationships of $\gamma v s . \tau_{50}$ and $\zeta$, which are corresponding to Eqs. (12) and (13), are obtained for the various "resistance ratio" $\gamma$ from the numerical integration under the consideration of adsorption term, and if these relations are diagramed previously, the analysis of experimental data becomes possible. A counting chart prepared in such a manner for the reduction with hydrogen at $550^{\circ} \mathrm{C}$ is shown in Fig. 2 . The data reported by Nakamura et al. ${ }^{17)}$ for fine Brazilian ores were quoted for the adsorption term in this calculation, and are shown in Fig. 3.

The numerical calculation was made by the graphical integration. From Eq. (5), $C_{i}-C_{o}$ becomes

$$
C_{i}-C_{o}=\left(1-\frac{r_{i}}{r_{o}}\right)\left(\frac{r_{i}}{r_{o}}\right) \cdot \gamma \cdot\left(C_{e}-C_{i}\right) \cdot f\left(C_{i}\right) \ldots \ldots
$$

Relations of $\left(r_{i} / r_{o}\right)$ vs. $C_{i}$ or $f\left(C_{i}\right)$ for a specified $\gamma$ are obtained by applying Eq. (15) to Fig. 3. Therefore, the graphical integration is possible when the relations obtained are substituted into Eq. (1) or (2).

In Fig. 2, $C_{i} *$ and $\lambda_{D} *$ are the maximum water concentration at the interface and the maximum relative diffusion resistance, i.e., the values of $C_{i}$ and 


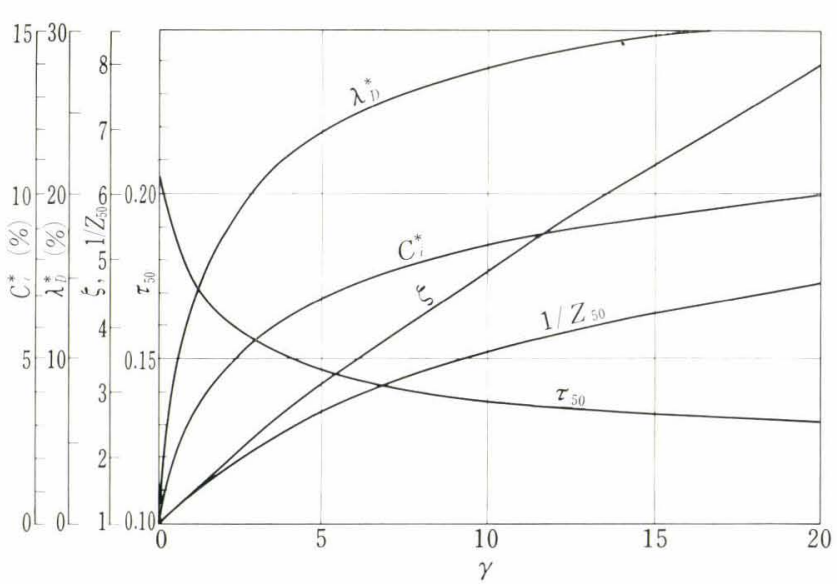

Fig. 2. A counting chart for the reduction data obtained at $550^{\circ} \mathrm{C}$

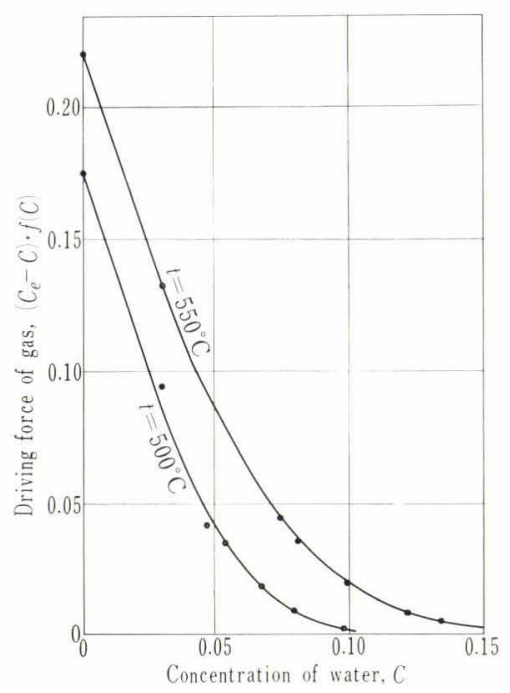

Fig. 3. Adsorption terms expressed in the form of driving force (experiment by Nakamura $\left.e t a l .{ }^{17}\right)$ )

$\lambda_{D} /\left(\lambda_{D}+\lambda_{R}\right)$ at $\left(r_{i} / r_{0}\right)=0.50$. In addition, $Z_{50}$ is the value of $Z$ at $50 \%$ reduction.

\section{Experiment}

\section{Sample, Apparatus, and Experimental Procedure}

Pellets used in the current work were prepared from the pulverized Brazilian ore ( -200 Tyler mesh). Brazilian ore is high quality hematite and the result of chemical analysis is shown in Table 1. The moistened ores were pelletized by a disk type pelletizer (25 cm in diameter). Pellets prepared were dried and fired in an electric resistance furnace at $1300^{\circ} \mathrm{C}$ for $1 \mathrm{hr}$ under air atmosphere. Furthermore, the samples used for the reduction experiments were previously

Table 1. Chemical composition of raw-ore used for pelletizing $(w t \%)$

\begin{tabular}{ccccccc}
\hline T. $\mathrm{Fe}$ & $\mathrm{Fe}^{++}$ & $\mathrm{Fe}^{+++}$ & $\mathrm{SiO}_{2}$ & $\mathrm{Al}_{2} \mathrm{O}_{3}$ & $\mathrm{~S}$ & $\mathrm{P}$ \\
\hline 68.76 & 0.55 & 68.20 & 0.60 & 0.67 & 0.003 & 0.024
\end{tabular}

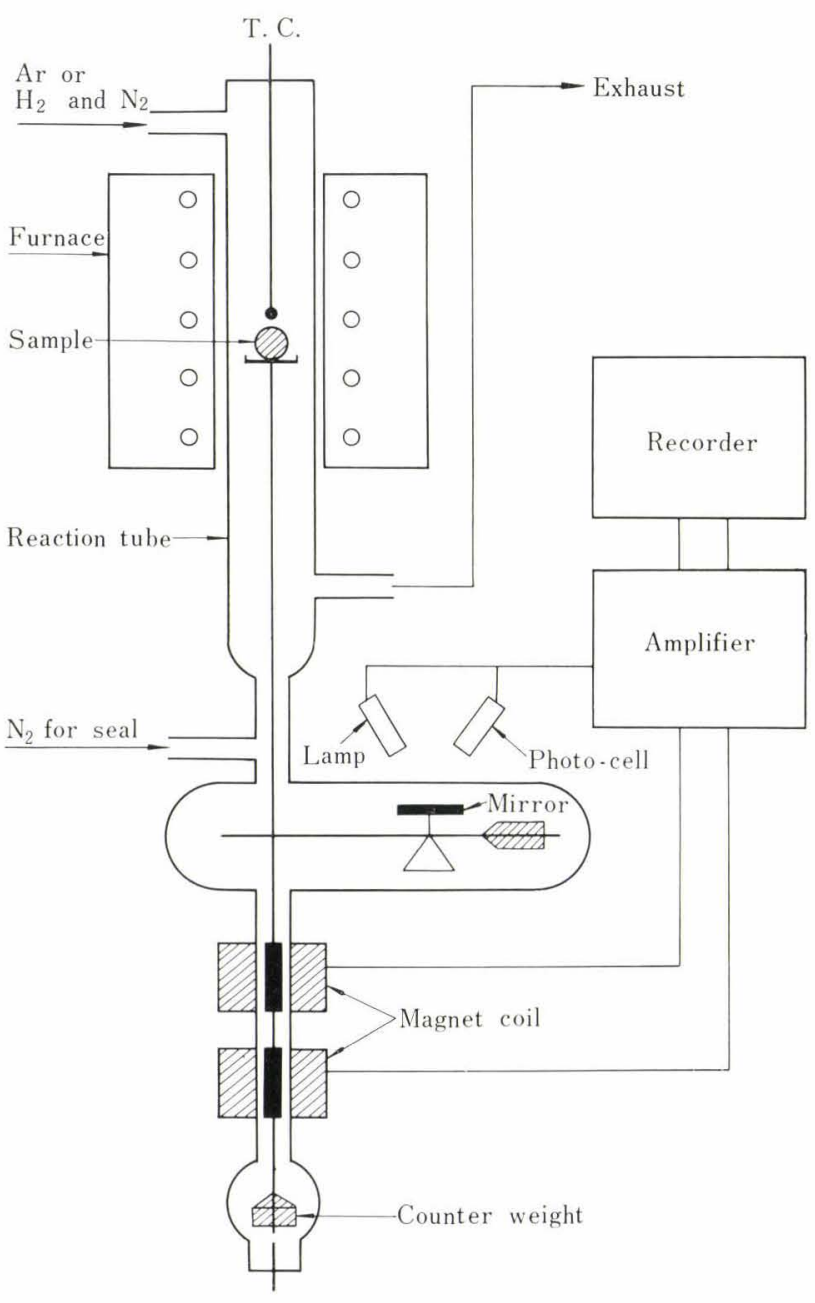

Fig. 4. A schematic diagram of the thermobalance

reduced to the composition of magnetite with a $\mathrm{H}_{2}-\mathrm{H}_{2} \mathrm{O}$ gaseous mixture at $550^{\circ} \mathrm{C}$. Pellets as prepared were also subjected for this experiment. The ranges of weight and diameter of samples were from 1.5 to $3.5 \mathrm{~g}$ and from 0.7 to $1.2 \mathrm{~cm}$, respectively. Porosities were about $28 \%$ for magnetite pellets (previously reduced) and about $20 \%$ for hematite pellets (as prepared). In order to examine the effects of pososity, the samples of hematite pellets having porosities of about 17, 24, and $30 \%$ were also made by adjusting the mixing ratio of raw powders and the rate of pelletizing. ${ }^{20)}$ Values of porosity were calculated from the apparent density measured by the mercury displacement method.

A schematic diagram of thermobalance used for the reduction experiment is shown in Fig. 4. The inclination of the arm due to the change in weight was detected by the aid of a photo-cell and a mirror mounted on the arm, and the inclination was revised by magnet coils. The weight change was automatically and continuously recorded by amplifying the current supplied for the coils. The maximum allowable weight change and the sensitivity of this thermobalance were 2.0 and $0.001 \mathrm{~g}$. The reaction tube was made of fused quartz ( $3 \mathrm{~cm}$ i.d.). The tube was heated with an electric furnace $(3 \mathrm{~kW})$. 
The quartz basket containing a sample pellet was suspended in the hot zone of reaction tube with a shaft connected to the arm of the thermobalance. The reaction temperature was measured by an AlumelChromel thermocouple placed just above the sample (within $5 \mathrm{~mm}$ ). The furnace was kept at a constant temperature within $1^{\circ} \mathrm{C}$ with a controller connected to the furnace. Before the reduction, a sample placed in the reaction tube was heated up to the reduction temperature under the flow of argon gas. After the sample was held at this temperature for a certain period of time, reducing gas was introduced to the system, and the weight change by the reduction was recorded. When the weight change ceased, that is, the reduction was completed, the reducing gas was stopped and argon was again introduced and the heating current was turned off. After the reaction tube was cooled to room temperature, the sample was taken out and the weight was checked again. The flow rate of reducing gas was always maintained above $5 \mathrm{Nl} / \mathrm{min}$ in order to eliminate the effects for reducing rate. Hydrogen, nitrogen and argon used were commercial grade cylinder gas.

\section{Results}

Final values of the weightloss by the reduction were equivalent to the reducible oxygen contents of pellets (29.5 wt \% in hematite pellets, $27.2 \mathrm{wt} \%$ in magnetite pellets) for all experiments. Therefore, the timeweightloss curves were regarded as the reducing curves.

A section of the magnetite pellet reduced partially with $\mathrm{H}_{2}$ at $550^{\circ} \mathrm{C}$ is shown in Photo. 1. In this photograph, it is obvious that the reduction was proceeding topochemically. The outer gray layer is reduced iron and the black core in center is unreduced magnetite. In the sections of hematite pellets, hematite phases disappeared even for the cases of very low degrees of reduction. For the analyses of data of hematite pellets, it was assumed that the reduction to magnetite was rapid and a part of the reducing curve above $11.1 \%$ in the overall degree of reduction was equivallent to the step from magnetite to iron.

The times required for 50 and $87.5 \%$ reduction were taken from the reducing curves and the data

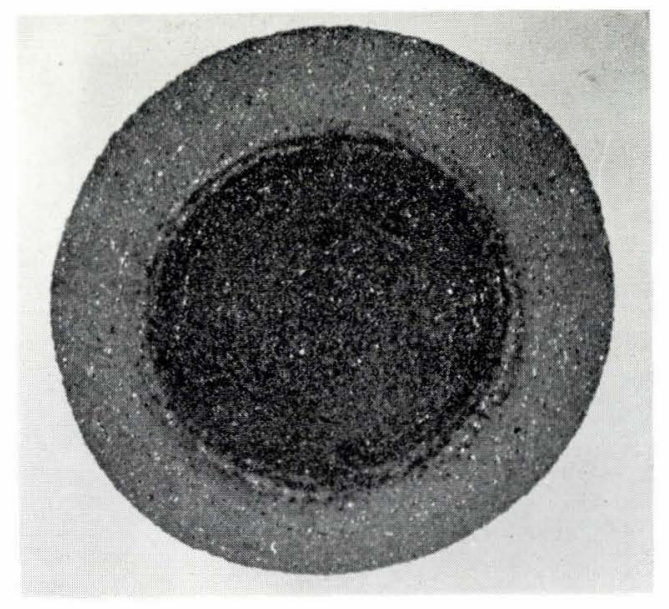

Photo. 1. Section of a partially reduced pellet at $550^{\circ} \mathrm{C}(\times 5.0)$ analyses were carried out by the method mentioned before. The example of analysis is as follows:

Example: run No. $M-1$ (a magnetite pellet, reduced with $\mathrm{H}_{2}$ at $550^{\circ} \mathrm{C}$ )

$w_{0}=3.36 \mathrm{~g}, r_{0}=0.612 \mathrm{~cm}, \varepsilon_{0}=0.297, \alpha=2.44 \times 10^{-4}$ $\mathrm{cm}^{3}$-pellet $/ \mathrm{cm}^{3}-\mathrm{H}_{2}$.

From the reducing curve, $\theta_{50}=14.2 \mathrm{~min}, \theta_{87.5}=49.0$ $\min$.

$$
\begin{aligned}
\therefore \quad \theta_{0} & =\theta_{87.5} \times 2=49.0 \times 2=98.0 \mathrm{~min} \\
\tau_{50} & =\theta_{50} / \theta_{0}=14.2 / 98.0=0.145
\end{aligned}
$$

From Fig. 2 at $\tau_{50}=0.145$

$$
\begin{gathered}
r=5.45, \quad \zeta=3.25, \quad C_{i}^{*}=7.05 \%, \quad \lambda_{D}^{*}=24.1 \%, \\
1 / Z=2.78 \\
\therefore \quad \theta_{0 \cdot C}=\theta_{0} / \zeta=98.0 / 3.25=30.2 \mathrm{~min}, \\
k_{c}=\frac{r_{o}}{\theta_{0} \cdot C \cdot \alpha}=\frac{0.612}{(30.2 \times 60)\left(2.44 \times 10^{-4}\right)}=1.38 \mathrm{~cm} / \mathrm{sec} \\
D_{e}=\frac{r_{o} \cdot k_{c}}{r}(1+1 / K)=\frac{(0.612)(1.38)}{(5.45)(0.220)}=0.704 \mathrm{~cm}^{2} / \mathrm{sec}
\end{gathered}
$$

Comparing $k_{c}$ with the value of single crystals, $k_{T h}$, reported by Nakamura et al. ${ }^{17)}$

$$
y=k_{c} \mid k_{T h}=1.38 / 0.128=10.8
$$

Comparing $D_{e}$ with gaseous diffusivity $D_{G}$,

$$
\delta=D_{e} / D_{G}=0.704 / 5.20=0.135
$$

The value of $y$ is over 10 in this example, meaning that the real area of reaction interface is larger than the apparent area. Therefore, the $k_{c}$ obtained from the analysis is the apparent value when it is assumed that the reaction area is equal to that of the spherical interface. In the porous pellet, the topochemical model is not perfectly realized but the reaction proceeds in the quasi-topochemical model which was mentioned by Schenck and Shulz. ${ }^{22}$ That is, some of the particle layers composed of the pellet are reacting simultaneously and the reacting zone is formed. However, the value mentioned above is not so large as to apply the topochemical model, since the surface area of one particle layer is $6 \times\left(1-\varepsilon_{0}\right)$ times larger than the apparent interface area.

The reducing curve of the foregoing example was recalculated by using $1 / Z=2.78$ and is shown in Fig. 5 where the value of $Z$ is taken as $Z_{50}$ for all degrees of reduction. In the case that the adsorption term is taken into account, the value of $Z$ for a specified $\gamma$ is independent of the degree of reduction and shows a nearly constant value. The recalculated and the experimental values show a good agreement.

Data for the reduction of magnetite pellets at $550^{\circ} \mathrm{C}$ with hydrogen were analyzed by considering the combined rate determining mechanism and the results obtained are shown in Fig. 6. The intraparticle diffusion coefficients $D_{e}$ and the chemical reaction constants $k_{c}$ show fixed values for the variations of the pellet weight $w_{0}$ within the experimental errors. Consequently, it is considered from Eq. (9), that $\gamma$ is pro- 


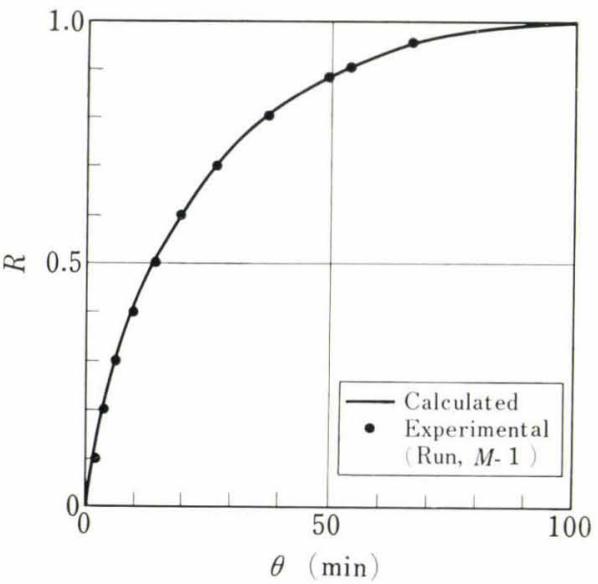

Fig. 5. Comparison of the reducing curve recalculated with the experimental value

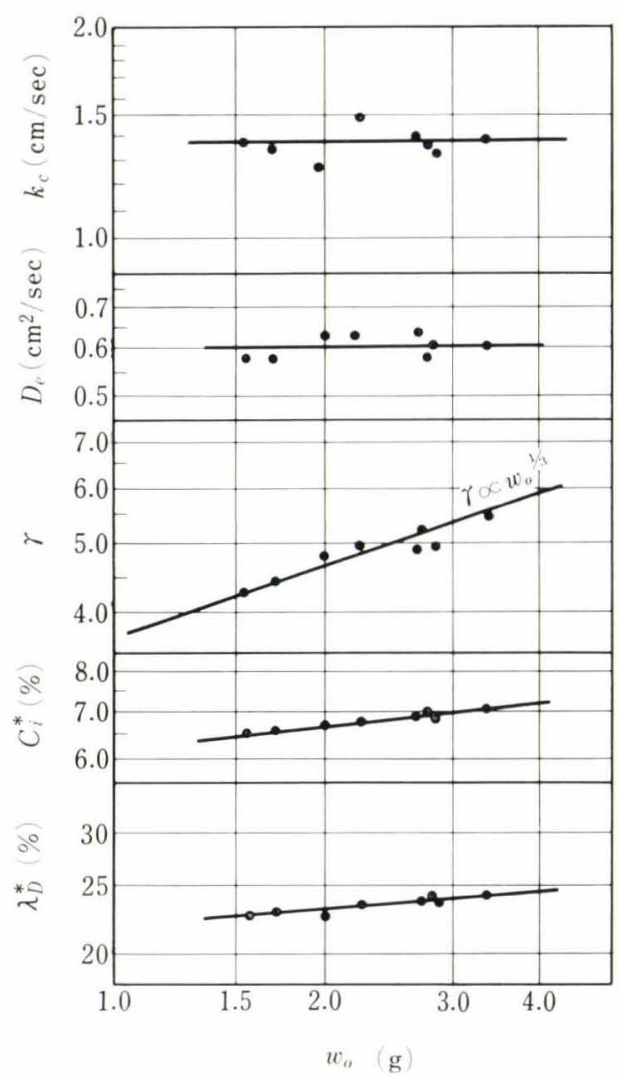

Fig. 6. Variations of $k_{c}, D_{e}, \gamma, C_{i}^{*}$, and $\lambda_{\nu}^{*}$, with pellet weight $w_{o}$ at $550^{\circ} \mathrm{C}$, pure $\mathrm{H}_{2}$ reduction

portional to the cube root of $w_{0}$. Increases of $C_{i}$ * and $\lambda_{D} *$ with $w_{0}$ are small.

The temperature dependences of $D_{e}$ and $k_{c}$ for the reduction of magnetite pellets are shown in Fig. 7. The relative change in $D_{e}$ with temperature agrees with that of gaseous diffusivity $D_{G}$. The diffusibility, $\delta=D_{e} / D_{G}$, is about 0.13 for $500^{\circ}$ and $550^{\circ} \mathrm{C}$. The activation energy of $k_{c}$ is smaller than that obtained by Nakamura et al. ${ }^{17)}$ for single crystals; it is about one half of the value reported by Nakamura. The values of magnifying factor of reaction surface $y$ are about 11 at $550^{\circ} \mathrm{C}$ and about 16 at $500^{\circ} \mathrm{C}$. This discrepancy

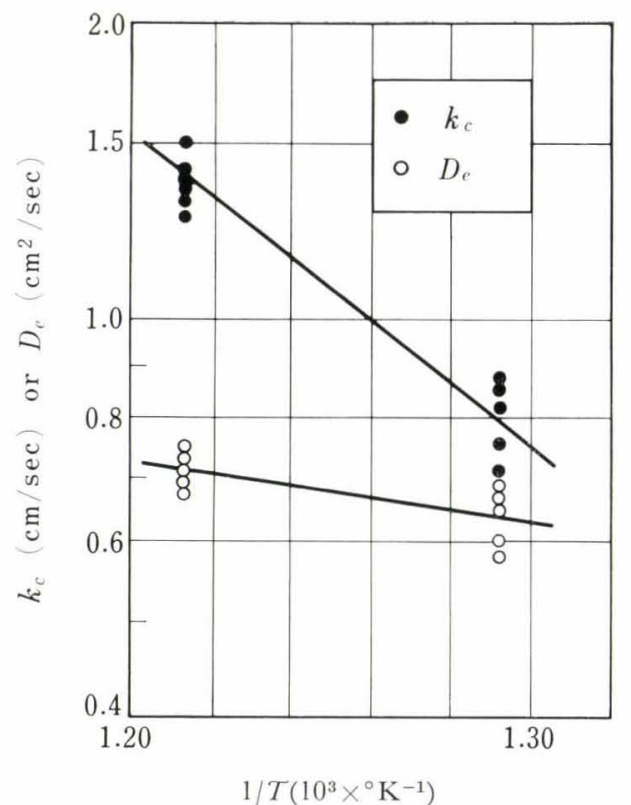

Fig. 7. Variations of $k_{r}$ and $D_{e}$ with temperature

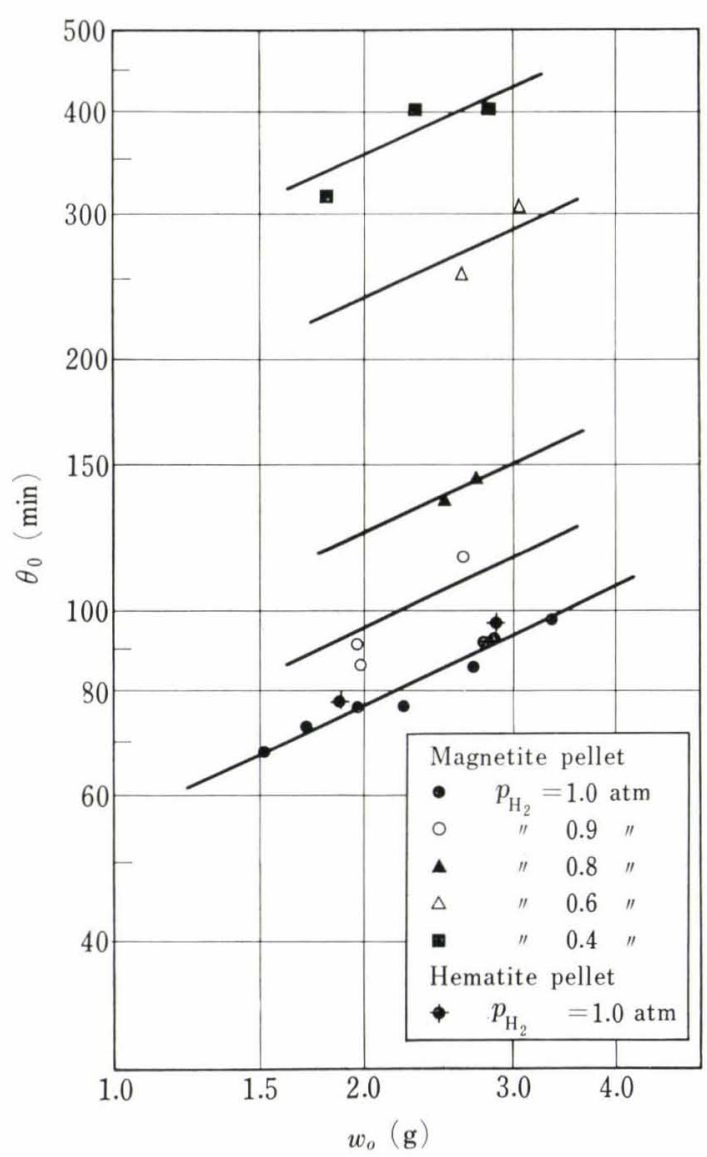

Fig. 8. Variations of the complete reduction time $\theta_{0}$ with $w_{0}$ at $550^{\circ} \mathrm{C}$

may be due to that the pellet is porous.

In the cases of hydrogen diluted with nitrogen, the times required for complete reduction $\theta_{0}$ are plotted in Fig. 8, in which the data of hematite pellet are added and those are almost in accord with that of magnetite pellet. The results of analyses by the combined rate determining mechanism nearly agreed with those of 


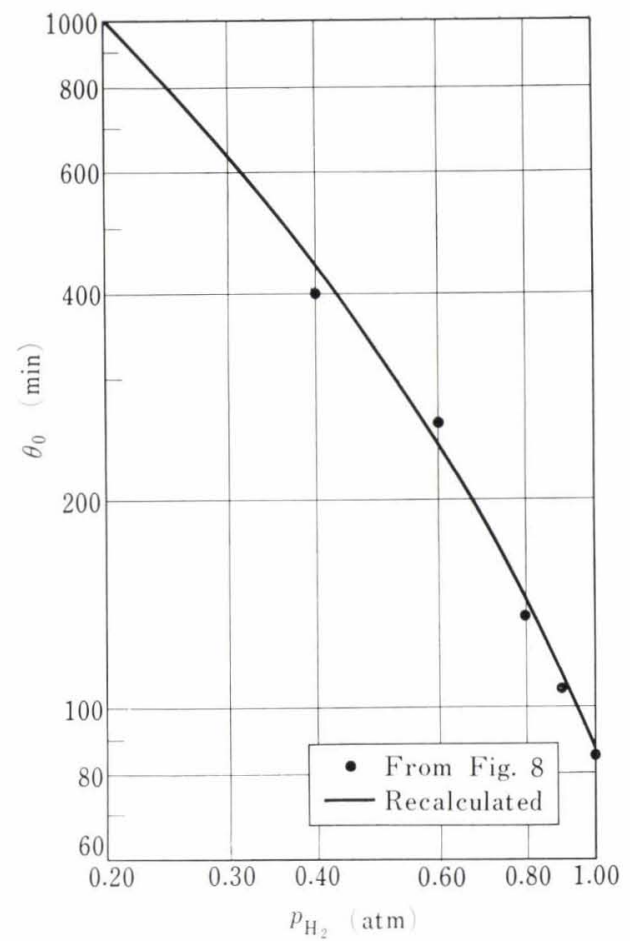

Fig. 9. Variation of $\theta_{0}$ with $\mathrm{H}_{2}$ partial pressure Reduction temp. : $550^{\circ} \mathrm{C}$ Diluent gas : $\mathrm{N}_{2}$ $w_{0}=2.50 \mathrm{~g}$ $\gamma=5.0$ $r_{0}=0.550 \mathrm{~cm}$ $k_{c}=1.37 \mathrm{~cm} / \mathrm{sec}$ $D_{e}=0.70 \mathrm{~cm}^{2} / \mathrm{sec}$

$\alpha=2.42 \times 10^{-4}$

Pure $\mathrm{H}_{2}$

magnetite pellet, and these were presented in the original paper. ${ }^{18)}$

The values of $\theta_{0}$ with $w_{0}=2.5 \mathrm{~g}$ obtained from Fig. 8 are plotted against the partial pressure of hydrogen in Fig. 9. From this figure, it is suggested that the reduction rate is not proportional to the hydrogen partial pressure and the degree of rate decreases more than that of the lowering of the partial pressure. Variations of $D_{e}$ and $k_{c}$ obtained from analyses by the combined rate control with the hydrogen partial pressure are shown in Fig. 10. Both of them decrease as the partial pressure decreases.

Next, $\theta_{0}$ in the reduction of the hematite pellets with 17,24 and $30 \%$ porosities at $550^{\circ} \mathrm{C}$ are shown in Fig. 11. Variations of $D_{e}$ and $k_{c}$ with porosity are shown in Figs. 12 and 13. These figures indicate that $D_{e}$ and $k_{c}$ are largely dependent on the porosity of pellet.

\section{Discussion}

\section{Validity of the Method of Analysis}

The topochemical proceeding of the reaction is assumed for the method of analysis proposed in the present study. In the strict sense, the quasi-topochemical model must however be applied to the porous pellet. Whether the topochemical model is applicable or not is determined by the relative effects of the intraparticle diffusion rate and the chemical reaction rate per unit volume of the pellet as seen in Photo. 1. According

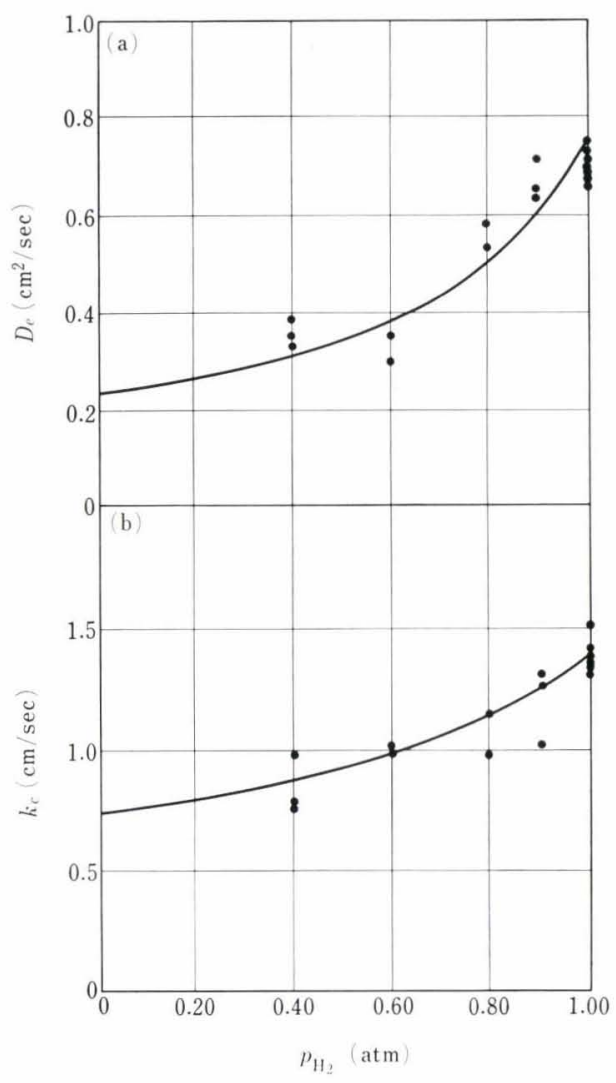

Fig. 10. Variations of $D_{e}$ and $k_{e}$ with $\mathrm{H}_{2}$ partial pressure at $550^{\circ} \mathrm{C}$

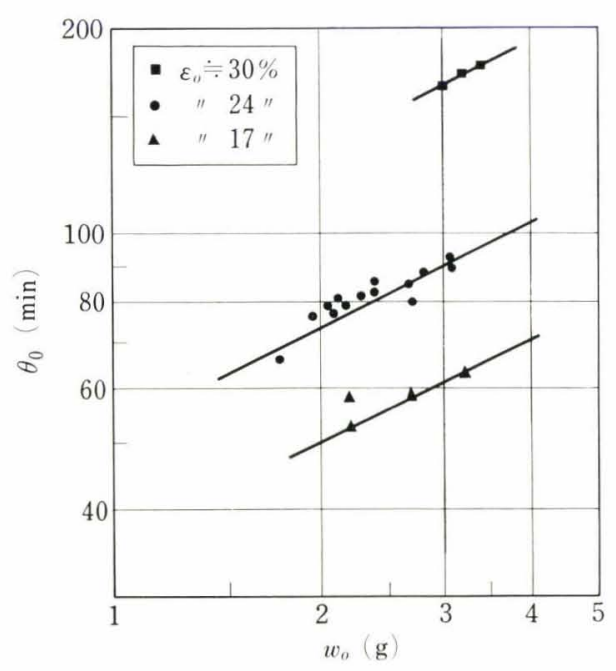

Fig. 11. Variations of $\theta_{0}$ with pellet porosity $\varepsilon_{o}$

to the temperature dependence of the intraparticle diffusivity and the rate constant of chemical reaction, it is possible to assume that more perfect topochemical model may be realized and the reacting interface may become sharper at higher temperatures. In lower temperatures, the discrepancy from the model is large and the reacting zone becomes broad. The similar fact was observed by Weisz and Goodwin ${ }^{23)}$ on the combustion of deposited carbon within the porous catalyst.

Bogdandy and Janke ${ }^{1)}$ showed that the thickness of 


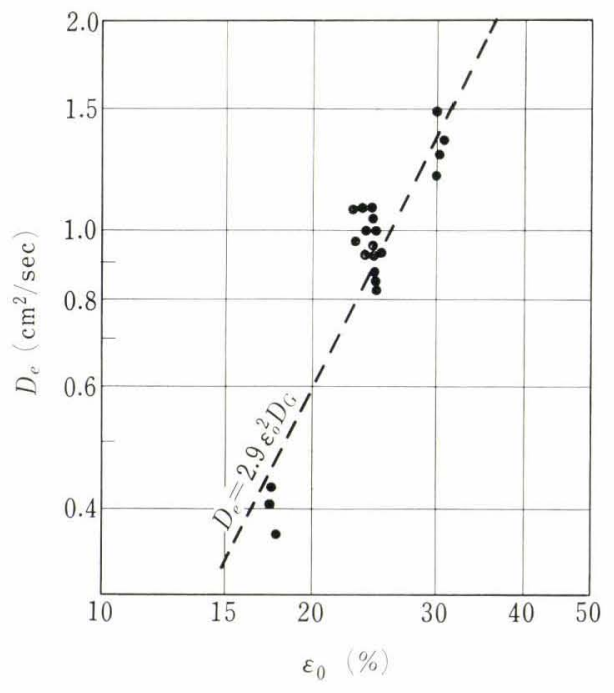

Fig. 12. Variation of $D_{e}$ with porosity

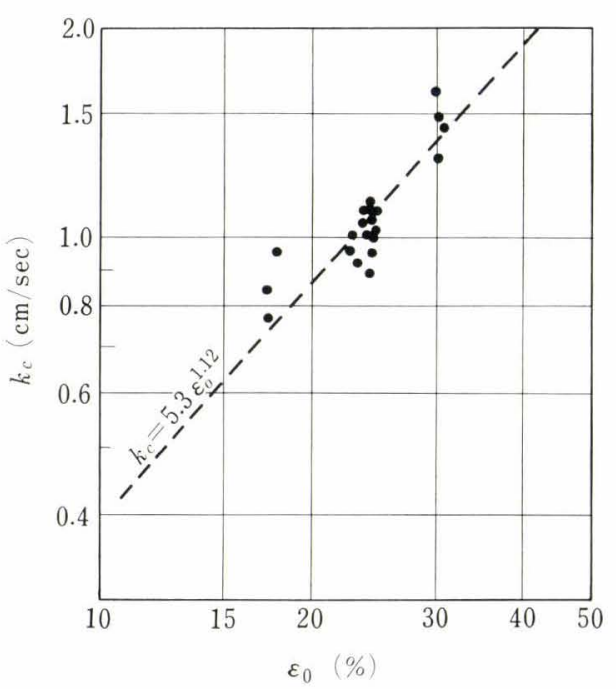

Fig. 13. Variation of $k_{c}$ with porosity

the reacting zone was about $3 \mathrm{~mm}$ when the pellet of $31 \mathrm{~mm}$ in diameter having a pososity of 19\% was reduced with hydrogen at $800^{\circ} \mathrm{C}$. The calculation based on the non-topochemical model which is accounting the diffusion in the reacting zone becomes more complicated. ${ }^{24), 25)}$ The topochemical model is generally applied in order to avoid this complication. ${ }^{1)-14}$ ) In many experiments, it is observed that the sections of partial reduced pellets show the topochemidal fashions $^{2)}{ }^{11)}$ as seen likewise in Photo. 1.

Mean radius of the particles composed of the pellets is presumed to be about $10 \mu$ from the size distribution of the raw powder, so that the specific surface area in the porous pellet may be very large. Therefore, the chemical reaction rate per unit volume becomes fairly great and the topochemical model will likely be realized. Moreover, in the reduction at temperature below $570^{\circ} \mathrm{C}$, the reduction proceeds only by the reaction between gas and solid, and there is no remarkable drop on the reducing rate at higher degree of reduction as seen in the hydrogen reduction at the

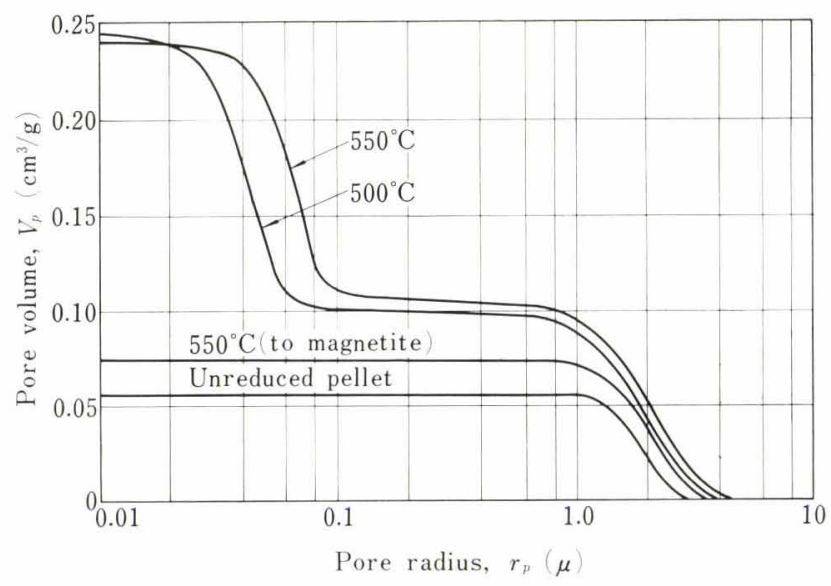

Fig. 14. Cumulative pore-size distribution curves of reduced pellets

temperatures between $800^{\circ}$ and $1000^{\circ} \mathrm{C}^{2}{ }^{26}$ ) From the values of the magnifying factor of reacting surface, the thickness of reacting zone is thought to be thin enough as compared with the pellet diameter. From this reason, it may be possible to adopt the topochemical model for the analysis.

However, the reaction and the diffusion are proceeding in parallel in the reacting zone. The magnifying factor of reacting surface is therefore affected by the variation of intraparticle diffusivity. Thiele's model which is used in the study of catalytic reaction may approximately be applied for this zone. Thiele's modulus $\phi=r_{o}\left(D_{e} / k_{c}\right)^{1 / 2}$ is nearly 50 according to the calculation for the reduction at $550^{\circ} \mathrm{C} .{ }^{19)}$ If $\phi>10$, the concentration gradient is proportional to $\phi$ in Thiele's model. Then if it is assumed that the thickness of the reacting zone is inversely proportional to the concentration gradient, the following relation would be derived for the magnifying factor. ${ }^{19}$

$$
\begin{aligned}
& y \propto\left(D_{e} / k_{T h}\right)^{1 / 2} \ldots \ldots \\
\text { Hence, } \quad & k_{e}=k_{T h} \cdot y \propto\left(k_{T h} \cdot D_{e}\right)^{1 / 2}
\end{aligned}
$$

Equation (16) shows that $k_{c}$ varies in proportion to the square root of $D_{e}$. If the variation of $D_{e}$ with temperature is small, the apparent activation energy of $k_{c}$ becomes about one half of that of $k_{T h}$. The temperature dependence of $k_{e}$ shown in Fig. 7 is explained from this concept.

In the present method, it is also assumed that the intraparticle diffusion is within the range of molecular diffusion. For this matter, the cumulative pore-size distributions of the reduced pellet, which were measured by the mercury penetration method, are shown in Fig. 14. ${ }^{27)}$ From the figure, it is found that the size of the pores formed by the reduction of magnetite to iron is several handreds $\mathrm{A}$ in the equivalent radius. This size is within the over-lapped range of molecular and Knudsen diffusions. However, the porous pellet contains macro-pores even in the as prepared state, the radius of which is larger than $1 \mu(10000 \AA)$ and the pore volume of pellet is corresponding to the porosity. It is therefore considered that the diffusion by the lat- 
ter, i.e., diffusion through the macro-pore, is predominant and the intraparticle diffusion could be treated as molecular diffusion.

As shown in Fig. 5, the reducing curve is well represented by the combined rate equation of intraparticle diffusion and chemical reaction. In order to check the values of $D_{e}$ obtained from the analyses, the gaseous diffusivities in the reduced pellets, which were cut out to the sphere segments, for $\mathrm{H}_{2}-\mathrm{N}_{2}$ systems were measured at room temperature by Wicke and Kaullenbach's procedure, ${ }^{28)}$ that is the measurement of the steady counter diffusion rates through the porous material at a constant total pressure. The diffusibilities $\delta$ were about 0.13 , and are agreed with those obtained by the reaction analyses. ${ }^{29)}$

If one analized the foregoing example without taking the adsorption term in account, the following results would be obtained. $k_{c}=1.18 \mathrm{~cm} / \mathrm{sec}, D_{e}=0.312 \mathrm{~cm}^{2} /$ sec, $C_{i} *=15.9 \%, \lambda_{D}^{*}=72.5 \%$. The value of $k_{c}$ is almost the same but $D_{e}$ is very small as compared with the case of taking the adsorption term in account. In this case, one would make wrong evaluation for the intraparticle diffusivity.

Here, let us consider the evaluation of the reducibility of porous pellet. In the reduction of a dense pellet at low temperature, the resistance of intraparticle diffusion can be neglected from Eq. (4), because there is no magnification of reacting area and $k_{c}$ becomes $k_{T h}$ owing to no penetration of the reducing gas into the unreduced core. Then, the reduction proceeds in the reaction control as it was demonstrated by McKewan. ${ }^{14)}$ Therefore, if the relative reducibility of the porous pellet is indicated by the ratio between the time required for complete reduction and that of the dense pellet of same size, the following expression is theoretically derived. ${ }^{18)}$

$$
\Phi=\theta_{T h} / \theta_{0}=\underset{\zeta\left(1-\varepsilon_{0}\right)}{y}
$$

The values of $\Phi$ obtained from Eq. (17) were about 4.5 at $550{ }^{\circ} \mathrm{C}$ and about 8.5 at $500^{\circ} \mathrm{C}$ in the reduction of magnetite pellets with $28 \%$ porosity. Those values approximately indicate the increases of reduction rate owing to the pellets porosity.

In the application of this method, the matter to be remarked is to use the data obtained under large gas velocities. If the resistance of the gas film cannot be neglected, the error of $\tau_{50}$ might be very large.

\section{Effects of Dituent Gas}

It was reported that the chemical reaction rate of reduction was proportional to the hydrogen partial pressure in the case that the reducing gas was diluted with an inert gas. ${ }^{17}$ ) However, Fig. 9 shows that the reduction rate is not proportional to the pressure. This phenomenon may be understood by considering the decrease in the intraparticle diffusivity at the presence of $\mathrm{N}_{2}$. From the diffusion equations for a three-component system, the following treatment is allowed.

In the case that the components $A$ and $B$ are diffusing in steady state as equimolal countercurrents and the component $C$ is a stagnant gas, the diffusion rates per unit cross section are given as follows by Toor, ${ }^{30)}$

$$
\mathcal{N}_{A}=-\mathcal{N}_{B}=\frac{P / \boldsymbol{R} L}{\left(1 / D_{A B}\right)-\left(1 / D_{B C}\right)} \cdot \ln \begin{aligned}
& x_{C, 1} \\
& x_{C, 0}
\end{aligned}
$$

where $D_{i j}$ is the diffusivity of two component system consisted of $i$ and $j, P$ the total pressure, $\boldsymbol{R}$ the gas constant, $L$ the length of diffusion path, $x_{c}$ the mole fraction of component $C$, and subscripts 0 and 1 indicate the points at both ends of the diffusion path. In this case, the following equation is yielded on the relation of concentrations.

$$
\begin{aligned}
& \left(1-\frac{D_{A B}}{D_{B C}}\right) \cdot\left(x_{A, 0}-x_{A, 1}\right)+\left(1-\begin{array}{c}
D_{A B} \\
D_{A C}
\end{array}\right) \cdot\left(x_{B, 0}-x_{B, 1}\right) \\
& =\ln \frac{x_{C, 1}}{x_{C, 0}}
\end{aligned}
$$

where $x_{A}$ and $x_{B}$ are mole fractions of components $A$ and $B$.

From Eq. (18), the steady state diffusion is not realized without the concentration gradient of the stagnant gas $C$, if $D_{A C} \neq D_{B C}$. This means that the concentration of $\mathrm{N}_{2}$ at the reacting interface are different from that of the outer surface during the reduction of the pellet with a $\mathrm{H}_{2}-\mathrm{N}_{2}$ mixture.

If Eq. (18) for the one-dimensional diffusion is applied to the diffusion in a spherical shell which is equivalent to the condition for pellet reduction, the diffusion rate is expressed by the following equation, using the effective diffusivity,

$$
n_{A}=-n_{B}=\begin{aligned}
& 4 \pi r_{o} r_{i} \\
& r_{o}-r_{i}
\end{aligned}\left(\begin{array}{c}
1 / D_{A C, e}-1 / D_{B C, e}
\end{array}\right) \ln \begin{aligned}
& x_{c, 1} \\
& x_{c, 0}
\end{aligned} \ldots
$$

Equation (19) can also be applied to the diffusion in a spherical shell if the diffusibilities are independent of the gas component.

Let us apply Eq. (19) to the $\mathrm{H}_{2}-\mathrm{H}_{2} \mathrm{O}-\mathrm{N}_{2}$ ternary system at $550^{\circ} \mathrm{C}$ and 1 atmosphere. In Eq. (19), components $A, B$, and $C$ correspond to $\mathrm{H}_{2}, \mathrm{H}_{2} \mathrm{O}$, and $\mathrm{N}_{2}$, respectively. When the mole fraction of $\mathrm{H}_{2} \mathrm{O}$ at the outer surface $x_{B, 0}$ is zero and the fractions of $\mathrm{N}_{2}$ at the surface $x_{C, 0}$ are $0.2,0.4 \cdots 1.0$, respectively, the relations between the mole fractions of $\mathrm{H}_{2} \mathrm{O}$ and $\mathrm{N}_{2}$ at the interface calculated from Eq. (19) are shown in Fig. 15, provided from ordinary calculations ${ }^{31), 32)}$ that $D_{A B}=$ 5.20, $D_{A C}=4.65$, and $D_{B C}=1.56 \mathrm{~cm}^{2} / \mathrm{sec}$ (in Fig. 15, regions indicated by dotted lines are not in existence). This figure shows that the relation of $x_{B, 1}$ and $x_{C, 1}$ can be expressed by a linear equation in $x_{B, 1}<0.20$. Slope of the straight line is given by ${ }^{19)}$

$$
a=-D_{A B} \frac{\left(1 / D_{A C}\right)-\left(1 / D_{B C}\right)}{1-\left(D_{A B} / D_{B C}\right)-\left(1 / x_{C, 1}\right)}
$$

As the difference between $x_{C, 0}$ and $x_{C, 1}$ is small in the region of $x_{B, 1}<0.20, x_{C, 1}$ in Eq. (21) may be substituted with $x_{C, 0}$. For the case of $C_{B, 0}=0$, the diffusion rate of water can be expressed as follows by using such a slope : ${ }^{19)}$

$$
n_{B}=\frac{4 \pi r_{o} r_{i}}{r_{0}-r_{i}} \cdot D_{A B, e}^{\prime} \cdot C_{B, 1}
$$




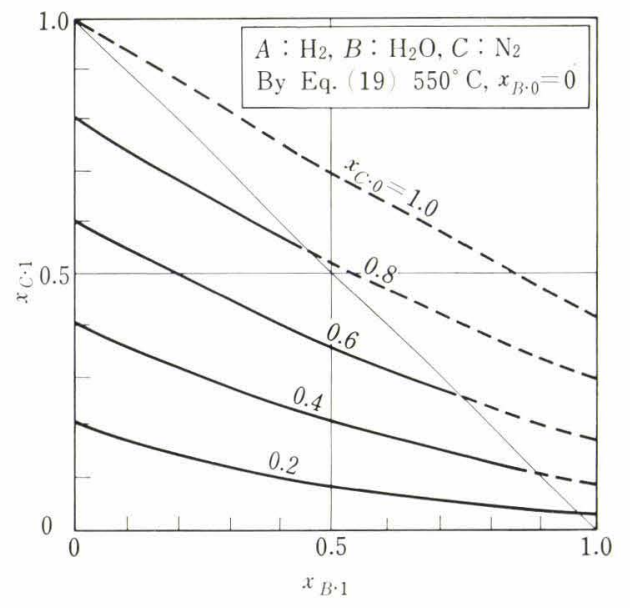

Fig. 15. Relation between mole fractions of components $B$ and $C$ at the interface

where $\quad D_{A B, e}^{\prime}=D_{A B, e}\left\{1-\left(1-\begin{array}{c}D_{A B} \\ D_{B C}\end{array}\right) \cdot x_{C, 0}\right\}^{-1}$

If the term of $D_{A B, e}^{\prime}$ is revised as the intraparticle diffusivity, Eq. (22) is identical with Eq. (1). Therefore, the analysis of reduction data obtained by $\mathrm{H}_{2}-\mathrm{N}_{2}$ gaseous mixtures becomes possible under the consideration of the combined rate determining mechanism. In other words, $D_{e}$ obtained by the method described in the preceeding chapter is equivalent to $D_{A B, e}^{\prime}$ and it varies with the $\mathrm{N}_{2}$ fraction $x_{C, 0}$ at the outer surface.

Next, let us consider the variation of $k_{c}$ with $x_{C, 0}$. If Eq. (23) is applied to Eq. (16) which was derived by assuming Thiele's model for the reacting zone, the following equation is obtained.

$$
k_{c}^{\prime}=k_{c}\left(\frac{D_{A B, e}^{\prime}}{D_{A B, e}}\right)^{1 / 2}=k_{c}\left\{1-\left(1-\frac{D_{A B}}{D_{B C}}\right) x_{C, 0}\right\}^{-1 / 2} \ldots
$$

The solid lines in Fig. 10 show the values calculated from Eqs, (23) and (24) provided that $D_{e}=0.750 \mathrm{~cm}^{2} /$ sec and $k_{c}=1.37 \mathrm{~cm} / \mathrm{sec}$ at $p_{H_{2}}=1.0 \mathrm{~atm}$, respectively. They coincide fairly well with the experimental. A solid line in Fig. 9 is the values of $\theta_{0}$ recalculated from the counting chart using the values of $D^{\prime}{ }_{c}$ and $k_{c}^{\prime}$ obtained from Eqs. (23) and (24). A good agreement with the experimental values is observed.

As described above, the effects of diluent gas are estimated by applying the concept of equimolal counter diffusion in three components to the intraparticle diffusion and Thiele's model to the reacting zone. McKewan ${ }^{33)}$ made a trial to explain the non-linear decreases of the reduction rate by considering the adsorption of diluent gas on the reacting surface. However, the explanation owing to the variation of the intraparticle duffusivity seems to be more appropriate, because the similar decreases are observed in case of using argon, which is not adsorbed chemically as a diluent gas. This fact had also been pointed out by Werner ${ }^{5)}$ and Spitzer et al. ${ }^{8)}$

According to Eq. (23), the intraparticle diffusivity varies from the value for $A-B$ system to that of $B-C$ system with the concentration of component $C$ in the outer bulk gas. Therefore, in case of reduction with $\mathrm{CO}-\mathrm{N}_{2}$ mixture, the decreases in the rate will not be so remarkable, because the difference between $D_{\mathrm{CO}_{-} \mathrm{CO}_{2}}$ and $D_{\mathrm{CO}-\mathrm{N}_{2}}$ is not so large.

\section{Effects of Porosity}

It seems that the study on the effective diffusivity through the porous material is not extended to estimate correct diffusibility or the tourtuosity factor from the structure of the material. Wakao and Smith ${ }^{34)}$ derived the equation for effective diffusivity of the bi-disperse material containing macropores and micropores from the model of random pore arrangement, and stated that the following equation might be used for the mono-disperse material which had only macropores being in the range of molecular diffusion,

$$
D_{e}=\varepsilon_{a}^{2} \cdot D_{G}
$$

where $\varepsilon_{a}$ is the void fraction of macropores.

In order to examine the equation mentioned above, the effective diffusivities of the unreduced pellets for $\mathrm{H}_{2}-\mathrm{N}_{2}$ system were measured at room temperature by Wicke-Kallenbach's procedure. The results coincided with the values multiplied by $0.9^{20)}$ of the right hand side of Eq. (25).

For the reduced pellet, if the macropore fraction is proportional to the porosity of the unreduced pellet, the intraparticle diffusivity would also be proportional to the square of the porosity of pellet from Eq. (25). A dotted line in Fig. 12 shows the equation.

$$
D_{e}=2.9 \varepsilon_{0}^{2} \cdot D_{G}
$$

The tendency for the variation of $D_{e}$ with $\varepsilon_{0}$ is the same. The magnifying coefficient is thought to be resulted from the increase of the macropore fraction and the contribution of the diffusion through the micropores formed by reduction.

Let us now consider the variation of $k_{c}$ with the porosity. If Thiele's model is applied to the reacting zone in the same manner as the effects of a diluent gas, the following equation would be derived by accounting the variations of $D_{e}$ and specific surface with $\hat{\varepsilon}_{0}{ }^{20)}$.

$$
k_{c} \propto \varepsilon_{0} /\left(1-\varepsilon_{0}\right)^{1 / 2} \fallingdotseq 1.36 \varepsilon_{0}^{1.12} .
$$

In this equation, the relationship of the most righthand side is realized in the range of $\varepsilon_{0}$ from 0.15 to 0.35. The relation between $\varepsilon_{0}$ and $k_{c}$ in experimental results is plotted in Fig. 13, and a dotted line shows the equation,

$$
k_{c}=5.3 \varepsilon_{0}^{1.12}
$$

The tendency of the variation with $\varepsilon_{0}$ shows a fairly good agreements with the theory. The value of $\theta_{0}$ recalculated from $D_{e}$ and $k_{c}$ in Eqs. (25) and (28) agreed very well with the experimental values, which were shown in the original paper, ${ }^{20)}$ and $\theta_{0}$ is inversely proportional to about 1.8 power of $\varepsilon_{0}$.

The effects of porosity are most important in the evaluation of the reducibility of porous pellets. However, it could be estimated in the experimental range 
of $\varepsilon_{0}$ from 0.15 to 0.35 , as mentioned above.

\section{Conclusions}

(1) A method of data analysis for the reduction of iron oxide pellets has been developed under the consideration of combined rate determining mechanism of the intraparticle diffusion and the chemical reaction involving the term of water vapor adsorption in it.

An assumption that the reaction proceeds topochemically and the times required for $50 \%$ and $87.5 \%$ reduction which is one-half of the complete reduction time in the combined rate determining mechanism are utilized in this method. By using these two reaction times obtained experimentally, the intraparticle diffusion coefficients and the chemical reaction constants are briefly calculated from such a counting chart as shown in Fig. 2 prepared by the numerical calculation employing the adsorption term.

(2) Porous pellets prepared from the pulverized Brazilian ore, high quality hematite, were reduced with hydrogen or hydrogen diluted with nitrogen at low temperatures $\left(500^{\circ}\right.$ and $550^{\circ} \mathrm{C}$ ) and the decreases in weight were measured with a thermobalance. Data obtained were analyzed by the method mentioned above and the effects of diluent gas and the pellet porosity were examined :

(i) In the strict sense, the reaction proceeds quasitopochemically in the porous pellet, but the topochemical model may approximately be applied for the analysis because the ratio between the intraparticle diffusion rate and the chemical reaction rate per unit volume of the pellet is large enough.

The values of intraparticle diffusivities obtained from the analyses agreed with the estimated values from gaseous diffusivity and diffusibility obtained by another experiment. Those temperature dependences were also the same with that of the gaseous diffusivity.

The chemical reaction constants obtained from the analyses were 10 times larger than those of single crystals. This discrepancy was owing to the magnification of the reacting surface. The temperature dependence of these constants was nearly half of that for single crystals and it was explained by applying Thiele's model to the reacting zone.

(ii) When the reducing gas $\mathrm{H}_{2}$ was diluted with $\mathrm{N}_{2}$, the reduction rate decreases more than that of $\mathrm{H}_{2}$ partial pressure. In this case, the variation of the intraparticle diffusion coefficients and the chemical reaction constants with $\mathrm{H}_{2}$ content can be estimated by applying the concepts of the equimolal counter diffusion for three component system to the intraparticle diffusion and Thiele's model to the reacting zone.

(iii) The reduction rate varied largely with the pellet porosities. The relative variations of the intraparticle diffusion coefficients and the chemical reaction constants are explained by applying the equation derived from the random pore model for the effective diffusivity of porous material.

\footnotetext{
Nomenclature

a : slope

$C_{0}, C_{e}, C_{i}, C_{i}^{*}$ : concentrations of $\mathrm{H}_{2} \mathrm{O}$ at outer surface, equili-
}

brium and interface and maximum concentration at interface, ( $\mathrm{g} \mathrm{mol} / \mathrm{cm}^{3}$ or fraction)

$D_{e}, D_{e}{ }^{\prime}, D_{G}$ : intraparticle diffusivities for reduction with $\mathrm{H}_{2}$ and $\mathrm{H}_{2}-\mathrm{N}_{2}$ mixture, and gaseous diffusivity, $\left(\mathrm{cm}^{2} / \mathrm{sec}\right)$

$k_{c}, k_{c}{ }^{\prime}, k_{T h} \quad$ : chemical reaction rate constants of pellet reduction with $\mathrm{H}_{2}$ and $\mathrm{H}_{2}-\mathrm{N}_{2}$ mixture and of reduction for single crystal, $(\mathrm{cm} / \mathrm{sec})$

$K \quad$ : equilibrium constant of reduction reaction

$K_{c} \quad$ : chemical reaction rate constant in rate per unit volume of pellet, ( $1 / \mathrm{sec})$

$L \quad$ : diffusion path, $(\mathrm{cm})$

$n \quad:$ diffusion rate in pellet, $(\mathrm{mol} / \mathrm{sec})$

$\mathcal{N} \quad$ : diffusion rate per unit cross section, $\left(\mathrm{mol} / \mathrm{cm}^{2}\right.$. sec)

$p_{\mathrm{H}_{2}} \quad$ : partial pressure of $\mathrm{H}_{2}$, (atm)

$P \quad$ : total pressure, (atm)

$r_{o}, r_{i}, r_{c} \quad$ : radii of pellet, reacting interface, and composed particle of pellet, $(\mathrm{cm})$

$R \quad$ : degree of reduction

$\boldsymbol{R} \quad$ : gas constant

$V, V_{C}, V_{D} \quad$ : overall reduction rate, chemical reaction rate, and diffusion rate of one pellet, $(\mathrm{mol} / \mathrm{sec})$

$w_{0} \quad$ : pellet weight, $(\mathrm{g})$

$x \quad$ : mole fraction of gas

$y \quad$ : magnifying factor of reaction surface

$Z \quad$ : relative ratio of $\tau$

$f\left(C_{i}\right) \quad$ : adsorption term of $\mathrm{H}_{2} \mathrm{O}$

$\gamma \quad$ : "resistance ratio " $-r_{0} k_{c}(1+1 / K) / D_{e}$

ò : diffusibility $=D_{e} / D_{G}$

$\varepsilon_{0}, \varepsilon_{a} \quad$ : porosity of unreduced pellet and fraction of macropore

: relative value $-\theta_{0} / \theta_{0} \cdot R$

$5 \quad$ : relative valir

$\theta_{0}, \theta_{50}, \theta_{87 \cdot 5}$ : times required for complete, $50 \%$ and $87.5 \%$ reduction, $(\mathrm{min})$

$\theta_{0} \cdot \mathrm{C} \quad$ : time required for complete reduction in chemical reaction control, $(\mathrm{min})$

$\lambda_{C}, \lambda_{D}, \lambda_{D}{ }^{*}$ : resistances of chemical reaction and intraparticle diffusion and relative maximum diffusion resistance, (sec/cm and \%)

$\begin{array}{ll}\tau & : \text { dimensionless reaction time }=\theta / \theta_{0} \\ \tau_{50,} \tau_{87,5} & : \text { values of } \tau \text { at } 50 \text { and } 87.5 \% \text { reduction }\end{array}$

$\tau_{50,} \tau_{87.5} \quad: \tau$ in chemical reaction control and in diffusion control

o : Thiele's modulus

$\Phi \quad$ : index for reducibility of pellet

\section{Subscripts}

$A, B, C \quad$ : components $A, B$, and $C$

$0,1 \quad$ : points of outer surface and interface of pellet

\section{REFERENCES}

1) L. Bogdandy and W. Janke: Z. Elektrochem., 61 (1957), 1146.

2) E. Kawasaki, J. Sanscrainte, and T. J. Walsh: A.I.Ch. E.J., 8 (1962), 48.

3) W. M. Mckewan: Trans. AIME, 218 (1960), 1.

4) N.J. Themelis and W. H. Gauvin: Trans. AIME, 227 (1963), 290; A. I. Ch. E.J., 8 (1962), 437.

5) N. A. Warner: Trans. AIME, 230 (1964), 163.

6) B. Seth and H. U. Ross: Trans. AIME, 233 (1965), 180.

7) H. W. Clair: Trans. AIME, 233 (1965), 1145.

8) R. H. Spitzer, F. S. Manning, and W. O. Philbrook: Trans. AIME, 236 (1966), 726.

9) M. A. Osman, F. S. Manning, and W. O. Philbrook: A. I. Ch. E. J., 12 (1966), 685. 
10) R. G. Olsson and W. M. McKewan: Trans. AIME, 236 (1966), 1518.

11) A. Moriyama, K. Ōtsu, and T. Imoto: Preprints of 3rd Reat. Eng. Symposium (Japan), (1963), 13.

12) K. Mori: Tetsu-to-Hagané, 50 (1964), 2559.

13) T. Ōtake, S. Tone, and S. Oda: Kagaku Kōgaku, 31 (1967), 71.

14) W. M. McKewan: Trans. AIME, 221 (1961), 140.

15) J. M. Quets, M. E. Wadsworth, and J. R. Lewis: Trans. AIME, 218 (1960), 545; 221 (1961), 1186.

16) K. Hedden and G. Lehman: Arch. Eisenhüttenw., 34 (1963), $887 ; 35$ (1964), 577.

17) Y. Nakamura, E. Tsunetomi, and S. Kondō: Preprints of 55 th J. I. M. Meetings (Toyama, Japan), (1964), 65.

18) Y. Hara, M. Sugata, T. Aida, and S. Kondō: J. Japan Inst. Metals, 31 (1967), 207.

19) Y. Hara, T. Aida, and S. Kondō: J. Japan Inst. Metals, 31 (1967), 987.

20) Y. Hara, T. Aida, and S. Kondō: J. Japan Inst. Metals, 32 (1968), 73.

21) K. B. Bischoff: Chem. Eng. Sci., 18 (1963), 711.
22) H. Schenck and H. P. Shulz: Arch. Eisenhüttenwe, 31 (1960), 691.

23) P. B. Weisz and R. D. Goodwin: J. Catalysis, 2 (1963), 397.

24) D. T. Lacey, J. H. Bown, and K. S. Basden: I \& EC Fundamentals, 4 (1965), 275.

25) S. Kasaoka and Y. Sakata: Kaguku Kōgaku, 30 (1966), 50.

26) M. Sugata, T. Aida, Y. Hara, and S. Kondō: J. Japan Inst. Metals, 31 (1967), 574.

27) S. Kondō, Y. Hara, M. Sugata, and M. Tsutiya: Tetsu-to Hagané, 53 (1967), 724.

28) E. Wicke and R. Kallenbach: Kolloid-Z., 97 (1941), 139.

29) S. Kondō, Y. Hara, and T. Aida: Tetsu-to-Hagané, 53 (1967), 1163.

30) H. L. Toor: A. I. Ch. E. J., 3 (1957), 198.

31) Society of Chemical Engineers, Japan: Kagaku Kōgaku Binran, (1958), 47, Maruzen, Tokyo.

32) S. Fujita: Kagaku Kōgaku, 28 (1964), 251.

33) W. M. Mckewan: J. Metals, 16 (1964), 781.

34) N. Wakao and J. M. Smith: Chem. Eng. Sci., 17 (1962), 825 . 